\title{
Busca Ativa de Possíveis Causas de Pirogenia em Pacientes Submetidos a Procedimentos Coronários Diagnósticos e Terapêuticos
}

\author{
Mônica Vieira Athanazio de Andrade ${ }^{1}$, Regina Silveira Silva ${ }^{2}$, Silvana Martins Dias Toni ${ }^{1}$, \\ Pedro Beraldo de Andrade ${ }^{1,2}$, Marden André Tebet ${ }^{1,2}$, André Labrunie ${ }^{1,2}$
}

\section{RESUMO}

Introdução: A reação pirogênica é desencadeada pela presença, na corrente sanguínea, de soluções contaminadas, infundidas no paciente, contendo endotoxinas ou produtos de degradação proteica. Apesar da baixa incidência, seu foco deve ser investigado e erradicado, pois essas ocorrências podem ser deletérias e refletem a qualidade do serviço prestado, já que a causa pode ser proveniente de fatores relacionados ao exame, às soluções utilizadas ou ao processo de esterilização. Método: Estudo prospectivo avaliando pacientes submetidos a procedimentos coronários percutâneos diagnósticos ou terapêuticos que desenvolveram reação pirogênica. Essa amostra foi comparada a um grupo controle de pacientes consecutivos que não apresentaram a referida intercorrência, sendo a possível causa pesquisada por meio do uso de um protocolo tipo check list. Resultados: No período de setembro de 2008 a fevereiro de 2009, de 1.026 procedimentos realizados, 25 (2,4\%) associaram-se a reações pirogênicas. Em todos os casos, a via de acesso utilizada foi a radial e os procedimentos caracterizaram-se pelo uso de materiais reprocessados. Foram investigados fatores técnicos, soluções utilizadas, condições de armazenamento e embalagem dos materiais, possíveis alterações no processo de trabalho dos prestadores de serviços internos, não sendo identificados fatores causais de pirogenia. A constatação da maior frequência de utilização de cateteres reprocessados no grupo que apresentou reações pirogênicas apontou a possibilidade de ocorrência de problemas relacionados à água de enxágue final. Após troca de componentes do filtro da água, cessaram os episódios de pirogenia nos exames. Conclusão: $A$ busca ativa por meio da elaboração de protocolos de investigação mostra-se uma ferramenta útil na identificação e erradicação de possíveis focos de pirogenia.

DESCRITORES: Cateterismo/efeitos adversos. Pirogênios. Endotoxinas. Água de enxágue. Microbiologia da água. Purificação da água. Reutilização de equipamento. Contaminação de equipamentos.

\footnotetext{
1 Santa Casa de Marília - São Paulo, SP, Brasil.

${ }^{2}$ Hospital do Coração de Londrina - Londrina, PR, Brasil.

Correspondência: Mônica Vieira Athanazio de Andrade. Praça Athos

Fragata, 25/1101 - Fragata - Marília, SP, Brasil - CEP 17501-220

E-mail: monicaathanazio@gmail.com

Recebido em: 10/3/2009 • Aceito em: 19/5/2009
}

\author{
ABSTRACT \\ Active Search of Pyrogen Reaction Sources in \\ Patients Undergoing Diagnostic and Therapeutic \\ Coronary Procedures
}

Background: Pyrogen reactions are triggered by intravenous injection of fluids or medications contaminated by bacterial endotoxins. Despite its low incidence, it must be investigated and eliminated, as it may be harmful to the patient and reflects the quality of the catheterization laboratory. Methods: Prospective study to evaluate patients undergoing diagnostic or therapeutic percutaneous coronary procedures who develop pyrogen reactions. This group was compared with a historical cohort of patients undergoing invasive procedures with no pyrogen reactions. The possible causes of pyrogen reactions were established based on the use of a check-list protocol. Results: From September 2008 to February 2009, $25(2.4 \%)$ of 1,026 percutaneous coronary procedures were associated to pyrogen reactions. Radial access was used in all cases, and the procedures were characterized by the use of recyclable materials. Technical aspects, fluids, storage and packaging conditions of the materials, possible changes in the routine of in-house service providers were investigated and the source of pyrogen reactions was not identified. The higher frequency of reusable catheters in the group with pyrogen reactions indicated a possible relationship with the water used to flush catheters in the final step of the sterilization process. Pyrogen reactions were no longer observed after replacement of a component of the water filter. Conclusions: The use of investigation protocols is an important tool in the identification and eradication of sources of pyrogen reactions.

DESCRIPTORS: Catheterization/adverse effects. Pyrogens. Endotoxins. Water microbiology. Water purification. Equipment reuse. Equipment contamination. 
A reação pirogênica é caracterizada por um conjunto de sinais e sintomas desencadeados pela presença, na corrente sanguínea, de soluções contaminadas por endotoxinas e produtos de degradação proteica infundidas no paciente. A manifestação clínica é aguda e caracterizada por febre, calafrios, tremores, hipotensão, cefaleia, dispneia, náuseas e redução na saturação de oxigênio ${ }^{1,2}$. O tratamento consiste na administração endovenosa de fármacos, com pronta melhora dos sintomas em grande parte dos casos.

Apesar de apresentar baixa incidência, o foco de pirogenia deve ser investigado e erradicado, pois suas manifestações clínicas podem ser deletérias ao paciente e refletem a qualidade do serviço prestado, uma vez que a causa pode ser proveniente de fatores relacionados ao exame, às soluções utilizadas ou ao processo de esterilização.

Com o objetivo de direcionar a busca e obter maior rapidez na detecção do fator causal, confeccionamos e aplicamos um protocolo para investigação de possíveis causas de pirogenia em pacientes submetidos a procedimentos coronários percutâneos diagnósticos ou terapêuticos.

\section{MÉTODO}

Estudo prospectivo, unicêntrico, avaliando 1.026 pacientes submetidos a procedimentos coronários percutâneos diagnósticos ou terapêuticos, no período de setembro de 2008 a fevereiro de 2009, dos quais $25(2,4 \%)$ desenvolveram reação pirogênica. A totalidade dos casos de pirogenia foi incluída no estudo, não havendo critérios de exclusão.

Como ferramenta de investigação, confeccionouse um protocolo do tipo check list, fundamentado nos possíveis fatores causais de reação pirogênica, que podem ter origem nos instrumentais e nas soluções utilizados durante o procedimento percutâneo ou na água de enxágue dos materiais da empresa de reprocessamento. Assim, foram documentados e avaliados sistematicamente todos os dados envolvidos nos procedimentos dos pacientes que desenvolveram reação pirogênica, como duração do exame, tempo de fluoroscopia, número de cateteres, fios-guia e introdutores reprocessados utilizados, soluções administradas aos pacientes, condições de armazenamento e embalagem dos materiais reprocessados, possíveis alterações no processo de esterilização dos campos cirúrgicos, das cubas rim e dos instrumentais realizado pela central de materiais e esterilização do hospital, bem como mudanças no processo de lavagem dos campos cirúrgicos pela lavanderia (Figura 1).

Buscando-se maior precisão e consistência dos dados, comparou-se a amostra que apresentou reação pirogênica a um grupo controle de pacientes que realizaram procedimentos coronários invasivos em intervalo de tempo semelhante. Uma vez que procedimen- tos coronários diagnósticos e terapêuticos diferem entre si em relação à duração média e à quantidade de materiais utilizados, notadamente quanto ao número de cateteres, os pacientes foram divididos em dois grupos distintos: procedimentos coronários diagnósticos e terapêuticos.

As variáveis categóricas foram expressas em frequência e porcentagem, comparadas com o teste do qui-quadrado. As variáveis contínuas foram expressas como média e desvio padrão, comparadas com o teste $t$ de Student ou teste exato de Fisher. Foram considerados estatisticamente significantes os resultados com valor de $\mathrm{P}<0,05$. Utilizou-se o programa SPSS, versão 12.0 (SPSS Inc., Chicago, Estados Unidos).

\section{RESULTADOS}

No período de setembro de 2008 a fevereiro de 2009, foram realizados 1.026 procedimentos coronários percutâneos, dos quais $25(2,4 \%)$ se associaram a reação pirogênica, sendo 21 coronariografias e quatro intervenções coronárias percutâneas. As manifestações clínicas foram constituídas predominantemente por tremores (Figura 2), sendo tratadas com administração de dipirona endovenosa, acrescida em quatro casos de hidrocortisona $200 \mathrm{mg}$ endovenosa e cloridrato de meperidina endovenoso em dois, com melhora imediata dos sintomas.

Todos os procedimentos foram realizados pelo acesso radial direito, utilizando-se cateteres pré-moldados de Judkins, e por três operadores diferentes. Para antissepsia e assepsia, utilizou-se rotineiramente clorexidina $2 \%$ degermante e alcoólica, sendo as demais soluções empregadas representadas por soro fisiológico $0,9 \%$, contraste iodado, xilocaína $2 \%$ sem vasoconstritor, heparina e monocordil, incluindo nitroglicerina nas intervenções coronárias percutâneas. De acordo com os laudos de análise microbiológica solicitados, foi constatada a esterilidade dos contrastes de baixa e alta osmolaridade. As demais soluções utilizadas estavam dentro do prazo de validade, íntegras e armazenadas adequadamente. As embalagens dos cateteres apresentavam-se íntegras e encontravam-se acondicionadas em armários fechados, livres de umidade e sujidade. O restante do material encontrava-se disposto em gavetas igualmente limpas. Em relação aos serviços prestados pelo hospital, incluindo lavagem, secagem, embalagem e esterilização de campos cirúrgicos, cubas rim e instrumentais utilizados nos procedimentos, não houve mudança na equipe, nas marcas, nas concentrações, nem nas diluições dos produtos, bem como nos tempos de exposição e de secagem. A troca do filtro da água utilizada na lavagem dos materiais atendia à periodicidade preconizada pela instituição.

Na Tabela 1 encontram-se expostos os dados técnicos dos procedimentos realizados em pacientes que apresentaram pirogenia, comparados aos do grupo 


\section{PROTOCOLO PARA INVESTIGAÇÃO DE POSSÍVEIS CAUSAS DE PIROGENIA EM PACIENTES SUBMETIDOS A PROCEDIMENTOS CORONÁRIOS DIAGNÓSTICOS E TERAPÊUTICOS}

\section{DADOS DO EXAME}

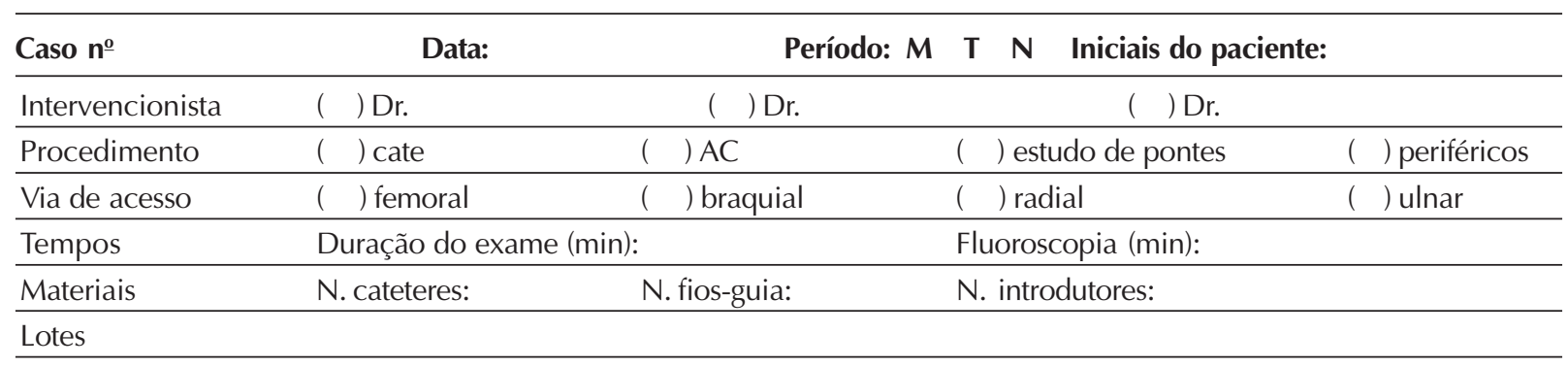

SOLUÇÕES UTILIZADAS NO PROCEDIMENTO

\begin{tabular}{|c|c|c|c|c|c|c|c|c|}
\hline \multirow[t]{2}{*}{ Solução } & \multicolumn{2}{|c|}{ Dentro da validade } & \multicolumn{2}{|c|}{ Laudo da empresa } & \multicolumn{2}{|c|}{ Aquecido } & \multirow[t]{2}{*}{ Lote } & \multirow[t]{2}{*}{ Empresa } \\
\hline & Sim & Não & Estéril & Não estéril & Sim & Não & & \\
\hline \multicolumn{9}{|l|}{ ( ) PVPI degermante } \\
\hline ( ) PVPI alcoólico & & & & & & & & \\
\hline \multicolumn{9}{|l|}{ ( ) Clorexidina degermante $2 \%$} \\
\hline \multicolumn{9}{|l|}{ ( ) Clorexidina alcoólica 0,5\% } \\
\hline \multicolumn{9}{|l|}{ Soro fisiológico $0,9 \%$} \\
\hline \multicolumn{9}{|c|}{$\begin{array}{l}\text { Osmolaridade do contraste } \\
(\quad) \text { baixa }(\quad) \text { isosmolaridade ( ) alta }\end{array}$} \\
\hline \multicolumn{9}{|l|}{ Cloridrato de lidocaína 2\% } \\
\hline Heparina sódica & & & & & & & & \\
\hline
\end{tabular}

\section{CONDIÇÕES DE ARMAZENAMENTO E EMBALAGENS DOS MATERIAIS}

\begin{tabular}{|c|c|c|c|c|c|c|}
\hline & \multicolumn{2}{|c|}{ Umidade } & \multicolumn{2}{|c|}{ Integridade } & \multicolumn{2}{|c|}{ Sujidade } \\
\hline & Sim & Não & Sim & Não & Sim & Não \\
\hline Locais de armazenamento & & & & & & \\
\hline Embalagens & & & & & & \\
\hline
\end{tabular}

\section{CENTRAL DE MATERIAIS E ESTERILIZAÇÃO INTERNA/LAVANDERIA}

\section{Alterações}

\begin{tabular}{|c|c|c|c|c|c|c|c|c|}
\hline & \multicolumn{2}{|c|}{ Produtos e quantidades } & \multicolumn{2}{|c|}{ Tempo de lavagem } & \multicolumn{2}{|c|}{ Tempo de enxágue } & \multicolumn{2}{|c|}{ Profissionais } \\
\hline & Sim & Não & Sim & Não & Sim & Não & Sim & Não \\
\hline Campos, cubas e pinças & & & & & & & & \\
\hline
\end{tabular}

\section{MANIFESTAÇÕES CLÍNICAS NO PACIENTE}

\begin{tabular}{|c|c|c|c|c|}
\hline Sinais e sintomas & $\begin{array}{l}(\quad) \text { febre } \\
(\quad) \text { cefaleia }\end{array}$ & $\begin{array}{l}\text { ( ) calafrios } \\
(\quad \text { ) dispneia }\end{array}$ & $\begin{array}{l}\text { ( ) tremores } \\
(\quad) \text { náuseas }\end{array}$ & $\begin{array}{l}\text { ( ) hipotensão } \\
\left(\quad \text { ) diminuição da Sat } \mathrm{O}_{2}\right.\end{array}$ \\
\hline Tempo & \multicolumn{2}{|c|}{ ) durante o procedimento: } & \multicolumn{2}{|c|}{ ) após o procedimento: } \\
\hline
\end{tabular}

\section{CONDUTA MÉDICA - TERAPIA MEDICAMENTOSA}

\begin{tabular}{llllll}
\hline Fármaco & $($ ) dipirona EV $\quad(\quad)$ hidrocortisona _ _ mg EV & $(\quad$ cloridrato de meperidina EV \\
\hline
\end{tabular}


controle de pacientes que não apresentaram tal reação. Os grupos não diferiram quanto às características clínicas, sendo $68 \%$ do sexo masculino, com média de idade de 60 anos.

Tanto em procedimentos diagnósticos como terapêuticos, não se observou diferença na duração total do procedimento entre aqueles que apresentaram ou não reação pirogênica. O tempo de fluoroscopia foi maior em pacientes submetidos a coronariografia que exibiram reação pirogênica $(P=0,07)$, denotando maior dificuldade técnica e possivelmente maior manipulação de instrumentais nesses casos.

Com relação à utilização de materiais reprocessados, a média do número de cateteres utilizados por procedimento diagnóstico foi de 2,3 no grupo pirogenia e de 1,9 no grupo controle $(P=0,0005)$. Os dois grupos apresentaram médias semelhantes do número de fios-guia utilizados $(1,1$ vs. 1,$1 ; P=1,0)$ e de introdutores (1) por procedimento.

O emprego do protocolo check list na análise dos dados contribuiu na busca e detecção dos possíveis fatores relacionados à ocorrência de reação pirogênica, facilitando a coleta, a organização e a interpretação dos achados.

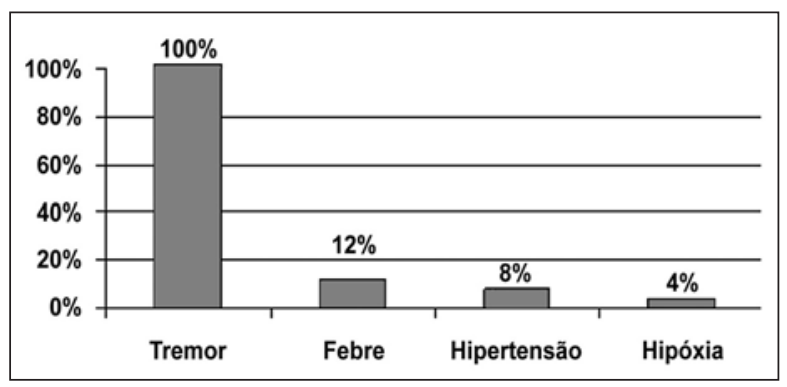

Figura 2 - Manifestações clínicas associadas às reações pirogênicas.

\section{DISCUSSÃO}

Em nossa casuística, a ocorrência de reação pirogênica em um período de seis meses foi infrequente $(2,4 \%)$. Por meio do emprego de um protocolo de investigação, foram avaliados os dados técnicos dos procedimentos, das soluções utilizadas, das condições de embalagem e armazenamento dos materiais, e das rotinas da central de materiais e esterilização e da lavanderia, não sendo identificados possíveis focos oriundos do setor, ou seja, provenientes da fase que se segue à filtração da água utilizada no enxágue de materiais pela empresa de esterilização externa.

Os achados demonstram que a duração do procedimento e o tempo de fluoroscopia não se associaram com a ocorrência de pirogenia. Em contrapartida, nos procedimentos diagnósticos, verificou-se, por meio de análise univariada, que o número de cateteres reprocessados se associou significativamente à ocorrência de reações pirogênicas, sugerindo a presença de endotoxinas em seu lúmen. Dados da literatura revelam que os materiais reprocessados podem causar reação pirogênica pelo fato de a água de enxágue desses materiais estar contaminada com bactérias ou endotoxinas.

Acredita-se que, ao serem reprocessados, os materiais possam ser contaminados com bactérias ou endotoxinas presentes na água de enxágue. Uma vez que a esterilização destrói os microrganismos viáveis, mas não suas endotoxinas, estas podem ser a causa das reações febris nos pacientes, mesmo que o produto esteja esterilizado ${ }^{3}$. Sabe-se que as endotoxinas, constituídas por complexos de alto peso molecular, consistem na mais importante fonte de pirógenos ${ }^{4}$. Recomenda-se que a água ou a solução à base de água utilizadas no enxágue dos materiais sejam livres de contaminação microbiana, uma vez que bactérias gram-negativas são capazes de sobreviver e crescer

TABELA 1

Comparação dos dados clínicos e relacionados ao procedimento entre o grupo que apresentou pirogenia e o grupo controle

\begin{tabular}{|c|c|c|c|c|c|c|}
\hline & \multicolumn{3}{|c|}{ Coronariografia } & \multicolumn{3}{|c|}{ ICP } \\
\hline & Pirogenia & Controle & $\mathbf{P}$ & Pirogenia & Controle & $\mathbf{P}$ \\
\hline Total (n) & 21 & 60 & - & 4 & 20 & - \\
\hline Sexo masculino (\%) & $14(67)$ & $35(58)$ & 0,6 & $4(100)$ & $16(80)$ & 1,0 \\
\hline Média de idade (anos) & $65,3 \pm 10,3$ & $61,2 \pm 9,4$ & 0,09 & $54 \pm 13$ & $60 \pm 13$ & 0,47 \\
\hline Duração do procedimento (min) & $17,1 \pm 9,94$ & $18,4 \pm 8,4$ & 0,56 & $35 \pm 11,5$ & $50,1 \pm 21,7$ & 0,19 \\
\hline Tempo de fluoroscopia (min) & $5,1 \pm 5,4$ & $3,6 \pm 2,2$ & 0,07 & $8,4 \pm 3,5$ & $14,2 \pm 8,1$ & 0,17 \\
\hline Cateter reprocessado $(\mathrm{n})$ & $2,3 \pm 0,7$ & $1,9 \pm 0,3$ & 0,0005 & $2,0 \pm 2,0$ & $2,3 \pm 0,7$ & 0,58 \\
\hline Fio-guia reprocessado (n) & $1,1 \pm 0,3$ & $1,1 \pm 0,3$ & 1,0 & $2,0 \pm 1,0$ & $1,3 \pm 0,7$ & 0,10 \\
\hline Introdutor reprocessado (n) & 1 & 1 & - & 1 & 1 & - \\
\hline
\end{tabular}

$\mathrm{ICP}=$ intervenção coronária percutânea; $\mathrm{n}$ = número; $\min =$ minutos. 
em água destilada ou deionizada ${ }^{5}$. Até mesmo gotículas de água remanescentes nos materiais, pela inibição da ação do óxido de etileno e outros processos de esterilização, podem predispor à sobrevivência e ao crescimento microbiano ${ }^{6}$.

Diferentes autores expuseram suas experiências e relatam a contaminação da água de enxágue como fator responsável pela ocorrência de reações pirogênicas $^{7-10}$. A revisão do processo de limpeza dos materiais reprocessados, com enfoque na utilização de água livre de pirógenos, constituiu-se na ação mais eficaz para a resolução do problema ${ }^{8,9}$. A água natural e destilada deve apresentar resultados negativos nos testes de endotoxina, em um nível de até 0,25 unidade de endotoxina (EU)/ml $\mathrm{ml}^{7}$. No Brasil, Duffy et al. ${ }^{10}$ investigaram 25 casos de pirogenia em Belo Horizonte, ocorridos após cateterização cardíaca, e detectaram, em média, 1.100 unidades formadoras de colônias (UFC) e endotoxinas na água deionizada utilizada na limpeza dos cateteres. Expondo-se a água a um filtro de $1 \mu \mathrm{m}$, a um ultrafiltro (em série) de 20.000 dáltons e a uma solução de hipoclorito de sódio a 5\%, obteve-se controle dos casos de pirogenia.

A análise microbiológica da água hospitalar de nosso serviço não foi realizada, pois, mesmo após sua utilização para limpeza mecânica e na lavadora ultrassônica, os materiais são posteriormente enviados a uma empresa de esterilização externa, onde sofrem novo processo de limpeza. Assim, é fundamental a avaliação da qualidade da água do último enxágue, em decorrência do risco da presença de endotoxina, recomendando-se sua monitoração quanto à qualidade química e microbiológica ${ }^{11}$.

Na totalidade das publicações supracitadas, identificou-se no sistema de suprimento de água a principal fonte de microrganismos e endotoxinas, demonstrando que o uso da água apirogênica no reprocessamento de materiais é fundamental para se evitar a ocorrência de pirogenia. Em paralelo à investigação com foco setorial, a empresa externa de reprocessamento e esterilização de materiais médico-hospitalares iniciou uma análise detalhada com rastreio das rotinas do serviço, na tentativa de identificar uma possível causa. Após a troca de um dos componentes do filtro de osmose reversa da água, não ocorreram novas reações pirogênicas, levando à hipótese de que a água de enxágue provavelmente seria o foco de contaminação com as endotoxinas.

Como limitações do estudo citaríamos: o fato de tratar-se de análise retrospectiva; número relativamente pequeno de pacientes; e ausência de análise microbiológica dos cateteres reprocessados para identificação inequívoca da presença de endotoxinas.

\section{CONCLUSÃO}

A identificação e a correção de possíveis fatores causais de reações pirogênicas não devem ser negligenciadas, pois, além de deletérias, comprometem a qualidade do serviço prestado ao paciente. A utilização de um protocolo de investigação tipo check list, fundamentado em busca ativa pelo enfermeiro, é uma ferramenta extremamente útil na pesquisa do possível foco de pirogenia, o qual pode estar associado à água utilizada no processo final de esterilização dos materiais médico-hospitalares.

\section{CONFLITO DE INTERESSES}

Os autores declararam inexistência de conflito de interesses.

\section{REFERÊNCIAS BIBLIOGRÁFICAS}

1. Sande MA, Levinson ME, Lukas DS, Kaye D. Bacteremia associated with cardiac catheterization. N Engl J Med. 1969;281(20):1104-6.

2. Lee RV, Drabinsky M, Wolfson S, Cohen LS, Atkins E. Pyrogen reactions from cardiac catheterization. Chest. 1973; 63(5):757-61.

3. Ribeiro SMCP. Reprocessamento de cateteres de angiografia cardiovascular após uso clínico e contaminados artificialmente: avaliação da eficácia da limpeza e da esterilização [dissertação de mestrado]. São Paulo: Escola de Enfermagem, Universidade de São Paulo; 2006.

4. Pinto TJA, Kaneko TM, Ohara MT. Controle biológico de qualidade de produtos farmacêuticos, correlatos e cosméticos. $2^{\text {a }}$ ed. São Paulo: Atheneu; 2003.

5. Jayabalan M. Sterilization and reprocessing of materials and medical devices: reusability. J Biomater Appl. 1995; 10(1):97-112.

6. Association for the Advancement of Medical Instrumentation (AAMI). Technical Information Report n. 12. Designing, testing, and labeling reusable medical devices for reprocessing in health care facilities: a guide for device manufactures. Arlington: AAMI; 1994.

7. Penna TC, Ferraz CA, Cassola MA. The presterilization microbial load on used medical devices and the effectiveness of hydrogen peroxide gas plasma against Bacillus subtilis spores. Infect Control Hosp Epidemiol. 1999;20(7):465-72.

8. Reyes MP, Ganguly S, Fowler M, Brown WJ, Gatmaitan BG, Friedman $C$, et al. Pyrogenic reactions after inadvertent infusion of endotoxin during cardiac catheterizations. Ann Intern Med. 1980;93(1):32-5.

9. Cookson ST, Nora JJ Jr, Kithas JA, Arduino MJ, Bond WW, Miller $\mathrm{PH}$, et al. Pyrogenic reactions in patients undergoing cardiac catheterization associated with contaminated glass medicine cups. Cathet Cardiovasc Diagn. 1997;42(1):12-8.

10. Duffy RE, Couto B, Pessoa JM, Starling C, Pinheiro S, Pearson $M L$, et al. Improving water quality can reduce pyrogenic reactions associated with reuse of cardiac catheters. Infect Control Hosp Epidemiol. 2003;24(12):955-60.

11. Takeiti MH, Graziano KU. Inovações tecnológicas no processamento da limpeza de artigos médico hospitalares. Revista SOBECC. 2000;5(1):12-7. 